\title{
$A$ Note on the Length of Proofs
}

\author{
Tsuyoshi Yukami
}

\section{§1. Introduction.}

Let $\boldsymbol{P A}$ be Peano arithmetic whose function symbols are ${ }^{\prime},+$ and $\cdot \boldsymbol{P A}^{*}$ is the corresponding system to $\boldsymbol{P A}$ with - replaced by a ternary predicate symbol $\boldsymbol{P}$ and the axiom saying that $P$ represents a function. The length of a proof is the maximal length of threads of proof. (For the definition of 'thread', see [16, p. 14].) For terms $s$ and $t, s<t$ is an abbreviation of $\exists x\left(s+x^{\prime}=t\right)$ for some appropriate bound variable $x$. For a natural number $n, \bar{n}$ denotes the term having" applied $n$ times to 0 .

In this paper the language of $\boldsymbol{L K}$ is that of $\boldsymbol{P A}^{*}$. For a natural number $m$, a formula $A$ and a set $\boldsymbol{S}$ of sentences, $\left.L \boldsymbol{K}\right|^{m} \boldsymbol{S} \rightarrow A$ means that there exists a proof, in $\boldsymbol{L K}$ with lenght $\leq m$, of a sequent $\Gamma \rightarrow A$ for some finite sequence $\Gamma$ of sentences in $\boldsymbol{S}$.

$S_{0}$ is the set of sentences of the following forms (1), (2), ,., (13).
(1) $\forall x(x=x)$
(2) $\forall x \forall y\left(x=y \supset x^{\prime}=y^{\prime}\right)$
(3) $\forall x \forall y \forall z \forall w(x=y \wedge z=w \supset x+z=y+w)$
(4) $\forall x \forall y \forall z(x=y \wedge y=z \supset x=z)$
(5) $\forall x \forall y \forall z(x=y \wedge x=z \supset y=z) \quad$ (6) $\forall x P(x, 0,0)$
(7) $\forall x \forall y \forall z\left(P(x, y, z) \supset P\left(x, y^{\prime}, z+x\right)\right)$
(8) $\forall x \forall y \forall z \forall w \forall u \forall v(x=y \wedge z=w \wedge u=v \wedge P(x, z, u) \supset \mathrm{P}(y, w, v))$
(9) $\bar{k}=\bar{m}+\bar{n}$, where $m$ plus $n$ is equal to $k$.
(10) $0=0+\cdots+0$
(11). $\forall x\left((x+\cdots+x)+\bar{m}=x^{\prime}+\cdots+x^{\prime}\right)$
(12) $\bar{n}+\stackrel{m}{n}+\bar{n}=\overline{m \times n})$

$$
P(\bar{m}, 0,0+\cdots+0) \wedge \forall x\left(P(\bar{m}, x, x+\cdots+x) \supset P\left(\bar{m}, x^{\prime}, x^{\prime}+\cdots+x^{\prime}\right)\right) \supset \forall
$$
$x P(\bar{m}, x, x+\cdots+x)$

For a primitive recursive function $f\left(n_{1}, \cdots, n_{\nu}\right), A_{f}\left(x_{1}, \cdots, x_{\nu}, x\right)$ denotes the standard formula of $\boldsymbol{P} \boldsymbol{A}^{*}$ which expresses the graph of $f$. For the precise definition of $A_{f}$, see [7, pp. 243-244].

Semi-RE-formulas are defined inductively as follows. 1. If $f\left(n_{1}, \cdots, n_{\nu}\right)$ is a

Shizuoka Gakuen College Surugadai, 4-1-1 Fujieda, 426 Japan 
primitive recursive function then $A_{f}\left(x_{1}, \cdots, x_{\nu}, 0\right)$ and $\neg A_{f}\left(x_{1}, \cdots, x_{\nu}, 0\right)$ are semiRE-formulas. 2. If $A$ and $B$ are semi-RE-formulas then so are $A \vee B$ and $A \wedge B$. 3. If $A(x)$ is semi-RE-formula then so is $\exists x A(x)$. 4. If $t$ is a term, $x$ does not occur in $t$ and $A(x)$ is a semi-RE-formula then $\forall x(x<t \supset A(x))$ is a semi-REformula.

$\Sigma_{1}$-formulas of $\boldsymbol{P} \boldsymbol{A}^{*}$ are defined by the same way as $\Sigma_{1}$-formulas of $\boldsymbol{P A}$ are defined in [6, p. 188]. I $\Sigma_{1}$ denotes the set of the universal closures of induction for $\Sigma_{1}$-formulas of $\boldsymbol{P A}^{*}$. $\quad \boldsymbol{S}_{1}$ is the set of sentences $\forall x \forall y(x=y \supset y=x), \forall x \forall y \forall$ $z \forall w(P(x, y, z) \wedge P(x, y, w) \supset z=w),(1),(2),(3),(4),(6),(7),(8)$ in the definition of $S_{0}$ and the transformations into the language of $\boldsymbol{P} \boldsymbol{A}^{*}$ of the first twelve sentences in the list of the axioms of $P^{-}$is [6, p. 188].

THEOREM. There is a sentence $A_{0}$ which satisfies the following properties (1), (2) and (3).

(1) $A_{0}$ is provable in the subtheory $I \Sigma_{1} \cup S_{1}$ of $P A^{*}$.

(2) for each primitive recursive function $f\left(n_{1}, \cdots, n_{\nu}\right)$, there exists a natural number $m$ such that

$$
\left.\boldsymbol{L K}\right|^{m} \boldsymbol{S}_{0} \cup\left\{A_{0}\right\} \rightarrow \mathrm{A}_{i}\left(\overline{\mathrm{n}_{1}}, \cdots, \overline{\mathrm{n}_{\nu}}, \overline{\mathrm{n}}\right)
$$

for each natural numbers $n_{1}, \cdots, n_{\nu}$ and $n$ with $f\left(n_{1}, \cdots, n_{\nu}\right)$ equal to $n$.

(3) For each semi-RE-formula $A\left(x_{1}, \cdots, x_{\nu}\right)$, there exists a natural number $m$ such that

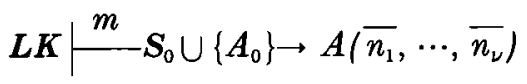

for each natural numbers $n_{1}, \cdots, n_{\nu}$ with $A\left(\overline{n_{1}}, \cdots, \overline{n_{\nu}}\right)$ true.

In the literature there are some sentences which are true but not provable in $\boldsymbol{P} \boldsymbol{A}^{*}$. The transformations into the language of $\boldsymbol{P \boldsymbol { A } ^ { * }}$ of $A_{q}(\bar{q})$ in [7, p. 208], Consis in $[7$, p. 210] and Paris-Harrington principle in $[14$, p. 1134] are typical examples of such sentences. Notice that the transformations of $A_{q}(\bar{q})$, Consis and ParisHarrington principle are of the formns $\forall x B_{1}(\bar{q}, x), \forall x B_{2}(\bar{r}, x)$ and $\forall x \forall y \forall z B$ ${ }_{3}(x, y, z)$, respectively, where $B_{1}(y, x), B_{2}(y, x)$ and $B_{3}(x, y, z)$ are semi-REformulas and $B_{1}(\bar{q}, \bar{n}), B_{2}(\bar{r}, \bar{n})$ and $B_{3}\left(\overline{n_{1}}, \overline{n_{2}}, \overline{n_{3}}\right)$ are true for each natural numbers $n_{1}, n_{2}, n_{3}$ and $n$. Hence the following corollary is immediate

COROLLARY. Let $A_{0}$ be any sentence satisfying the property (3) in Theorem. Then there is a natural oumber $m$ such that $\left.L K\right|^{m} \boldsymbol{S}_{0} \cup\left\{A_{0}\right\} \rightarrow B_{1}(\bar{q}, \bar{n}) \wedge B_{2}(\bar{r}$, $\bar{n}) \wedge B_{3}\left(\overline{n_{1}}, \overline{n_{2}}, \overline{n_{3}}\right)$ 
for each natural numbers $n_{1}, n_{2}, n_{3}$ and $n$.

Note that the sentences $(1),(2), \cdots,(8)$ in the definition of $\boldsymbol{S}_{0}$ are provable in $\boldsymbol{P A}^{*}$ and all sentences of the form (13) are provable in $\boldsymbol{P} \boldsymbol{A}^{*}$ with length 1 . Further note that all equations of the forms (9) and (10) are provable in $P A^{*}$ with length $\leq$ 25 (see $[15]$ and Fact 4 in [17]). Hence the following problem is very interesting.

PROBLEM. Is there a natural number $m$ such that all equations of the forms (11) and (12) are provable in $\boldsymbol{P A}\left(\boldsymbol{P} \boldsymbol{A}^{*}\right)$ with length $\leq m$ ?

(In the introduction of [17] we proposed this problem and pointed out that the affirmative answer to the probllem implies the negative answer to Kreisel's conjecture. For the literature related to Kreisel's conjecture, see [1], [2], [8], [9], references in $[\mathbf{9}],[12],[13],[18]$ and [19].)

In $\S 2$ we shall prove Theorem. In $\S 3$ we shall give three remarks. In the first remark we shall point out a relation between Theorem in [18] and the proof of Theorem in $\S 2$. The purpose of this remark is to explain that Theorem in [18] shows an importance of 'detour' in a proof for making the length of the proof short. The second remark is to explain that Theorem shows a proof-theoretic meaning of MRDP theorem. (MRDP theorem is the theorem asserting that every recursive enumerable predicate is diophantine.) The last remark is related to the $k$ provability problem of $\boldsymbol{P A}$. (For the literature related to the k-provability problems of $L K$ and $P A$, see [3], [5], [8], [9], [11] and [13].)

\section{§2. Proof of Theorem.}

First of all we define E-formulas inductively as follows. 1. Atomic formulas are E-formulas. 2. If $A$ and $B$ are E-formulas then so are $A \vee B$ and $A \wedge B . \quad 3$. If $A(x)$ is an E-formula then so is $\exists x A(x)$.

$A_{1}, A_{2}, \cdots, A_{8}$ are the sentences (1), (2), $\cdots,(8)$ in the definition of $\boldsymbol{S}_{0 .} \varphi(m)$ and $\phi(k, m)$ are the functions defined as follows. 1. $\varphi(0)$ is 4.2 . $\varphi(m+1)$ is $\varphi(m)+\mathrm{m}^{2}+5 \times m+18$. 3. $\phi(0, m)$ is $\max \left(\varphi(m)+m^{2}+10 \times m+27,3 \times m+99\right)$. 4. $\psi(k+1, m)$ is $\psi(k, m)+m^{2}+16 \times k^{2}+4 \times k \times m+36 \times k+m+3$.

\section{LEMMA 1.}

(1) Let $m$ be the number of function symblos in a term $t\left(x_{1}, \cdots, x_{\nu}\right)$. For each natural numbers $n_{1}, \cdots, n_{\nu}$; if $t\left(\overline{n_{1}}, \cdots, \overline{n_{\nu}}\right)$ represents a natural number $n$, then $\Gamma$, $A_{4}, A_{3}, A_{2}, A_{1} \rightarrow \bar{n}=t\left(\overline{n_{1}}, \cdots, \overline{n_{\nu}}\right)$ is provable in $L K$ with length $\leq \varphi(m)$ for some finite sequence $\Gamma$, with length $\leq m$, of equations of the form (9) is $\boldsymbol{S}_{\mathbf{0}}$.

(2) $\bar{n}+\cdots+\bar{n}=(\overline{n \times m}), \quad P(\bar{m}, 0,0+\cdots+0) \wedge \forall x(P(\bar{m}, x, x+\cdots+x) \supset$ 
$\left.P\left(\bar{m}, x^{\prime}, x^{\prime}+\cdots+x^{\prime}\right)\right) \supset \forall x P(\bar{m}, x, x+\cdots+x), \quad 0=0+\cdots+0, \quad \forall x((x+\cdots+x)+$ $\left.\bar{m}=x^{\prime}+\cdots+x^{\prime}\right), A_{8}, A_{7}, A_{6}, A_{1} \rightarrow P(\bar{m}, \bar{n}, \overline{m \times n})$ is provable in LK with length 50 .

(3) Let $m$ be the number of function symbols in an E-formula $B\left(x_{1}, \cdots, x_{\nu}\right)$ and $k$ be that of logical symbols in it. For each natural numbers $n_{1}, \cdots, n_{\nu}$; if $B\left(\overline{n_{1}}, \cdots, \overline{n_{\nu}}\right)$ is true then $\Gamma, A_{8}, A_{7}, \cdots, A_{1} \rightarrow B\left(\overline{n_{1}}, \cdots, \overline{n_{\nu}}\right)$ is provable in $\mathbf{L K}$ with length $\leq \psi(k, m)$ for some finite sequence $\Gamma$, with length $\leq m+4 \times(k+1)$, of sentences of the forms (9), (10), (11), (12) and (13) in $\boldsymbol{S}_{0}$.

\section{Proof.}

(1) is proved by induction on the number of function symbols in $t\left(x_{1}, \cdots, x_{\nu}\right)$.

(2) $0=0+\cdots+0, A_{8}, A_{6}, A_{1} \rightarrow P(\bar{m}, 0+\cdots+0)$ is provable in $L K$ with length 16. $\forall x\left((x+\cdots+x)+\bar{m}=x^{\prime}+\cdots+x^{\prime}\right), A_{8}, A_{7}, A_{1} \rightarrow P(\bar{m}, a, a+\cdots+a) \supset P\left(\bar{m}, a^{\prime}\right.$, $\left.a^{\prime}+\cdots+a^{\prime}\right)$ is provable in $L K$ with length 24 . Hence $P(\bar{m}, 0,0+\cdots+0) \wedge \forall$ $x\left(P(\bar{m}, x, x+\cdots+x) \supset P\left(\bar{m}, x^{\prime}, x^{\prime}+\cdots+x^{\prime}\right)\right) \supset \forall x P(\bar{m}, x, x+\cdots+x), 0=0+\cdots+$ $0, \forall x\left((x+\cdots+x)+\bar{m}=x^{\prime}+\cdots+x^{\prime}\right), A_{8}, A_{7}, A_{6}, A_{1} \rightarrow P(\bar{m}, \bar{n}, \bar{n}+\cdots+\bar{n})$ is provable in $\boldsymbol{L K}$ with length 32 and so $\bar{n}+\cdots+\bar{n}=(\overline{n \times m}), \mathrm{P}(\bar{m}, 0,0+\cdots+0) \wedge \forall$ $x\left(P(\bar{m}, x, x+\cdots+x) \supset P\left(\bar{m}, x^{\prime}, x^{\prime}+\cdots+x^{\prime}\right)\right) \supset \forall x P(\bar{m}, x, x+\cdots+x), 0=0+\cdots+$ $0, \forall x\left((x+\cdots+x)+\bar{m}=x^{\prime}+\cdots+x^{\prime}\right), A_{8}, A_{7}, A_{6}, A_{1} \rightarrow P(\bar{m}, \bar{n}, \overline{m \times n})$ is provable in $L K$ with length 50 .

(3) is proved by induction on the number of logical symblos in $B\left(x_{1}, \cdots, x_{\nu}\right)$. In the basis step, use (1) and (2).

Now Theorem is immediate from (3) in Lemma 1, the following lemma and the fact that each provable sentence in $\boldsymbol{P A} \boldsymbol{A}^{*}$ is true.

LEMMA 2. There is a sentence $A_{0}$ which satisfies the following properties (1), (2) and (3).

(1) $A_{0}$ is provable in the subtheory $I \Sigma_{1} \cup \boldsymbol{S}_{1}$ of $\boldsymbol{P A}$.

(2) For each primitive recursive function $f\left(n_{1}, \cdots, n_{\nu}\right)$, there exists an $E$-formula $B\left(x_{1}, \cdots, x_{\nu}, x\right)$ such that $A_{0} \rightarrow \forall x_{1} \cdots \forall x_{\nu} \forall x\left(A_{f}\left(x_{1}, \cdots, x_{\nu}, x\right) \equiv B\left(x_{1}, \cdots, x_{\nu}, x\right)\right)$ is provable in $\mathbf{L K}$.

(3) For each semi-RE-formula $A\left(x_{1}, \cdots, x_{\nu}\right)$, there exists an $E$-formula $B\left(x_{1}, \cdots\right.$, $\left.x_{\nu}\right)$ such that $A_{0} \rightarrow \forall x_{1} \cdots \forall x_{\nu}\left(A\left(x_{1}, \cdots, x_{\nu}\right) \equiv B\left(x_{1}, \cdots, x_{\nu}\right)\right)$ is provable in $L K$.

Proof. This lemma is a careful formalization of the proof given in [4] of MRDP Theorem. For details, see $\S 4$ and $\S 6$ is [6] with the following two changes added. (1) Since we need the equivalence on the last line in [6, p. 198], we must work in $I \Sigma_{1}$ instedad of $I \Sigma_{0}+$ exp. Notice that exp is provable in $I \Sigma_{1}$. Further notice that $\forall x_{1} \cdots \forall x_{\nu} \exists ! x A_{f}\left(x_{1}, \cdots, x_{\nu}, x\right)$ is provable in $I \Sigma_{1}$ for each primitive recursive function $f\left(n_{1}, \cdots, n_{\nu}\right)$. Hence $\neg A_{f}\left(x_{1}, \cdots, x_{\nu}, 0\right) \equiv \exists x A_{f}\left(x_{1}, \cdots, x_{\nu}, x^{\prime}\right)$ is 
provable in $I \Sigma_{1}$. (2) To replace $I \Sigma_{1}$ by a single sentence we use the truth definition for $\Sigma_{1}$-formulas insted of that for $\Sigma_{0}$-formulas.

\section{§3. Remarks.}

REMARK 1. It seems that there exists a relation between Theorem in [18] and the froof of Theorem in $\S 2$. Though the relation is not rigorous, the author wants to explain it.

Roughly speaking, Theorem in [18] asserts that we can divide a short (in contrast with $n$ ) proof in $\boldsymbol{P A}$ of $A(\bar{n})$ into a proof in $\boldsymbol{L K}$ and 'purely numbertheoretic' proof. The former proof proves $\forall x(B(x) \supset A(x))$ for some formula $B(x)$. The latter proof proves $B(\bar{n})$ and the length of it is short in contrast with $n$. For more precise details, see [18].

In the proof of Theorem in $\S 2$, we gave a short proof of true semi-RE formula $A(\bar{n})$ in the following manner. First we found an E-formula $B(x)$ and gave a proof in $L K$ of $A_{0} \rightarrow \forall x(B(x) \equiv A(x))$. Second we gave a short proof in $L K$ of $\Gamma \rightarrow B(\bar{n})$ for some finite sequence $\Gamma$ of sentences in $S_{0}$. Finally, combining the above two proofs, we gave a short proof in $L K$ of $\Gamma, A_{0} \rightarrow A(\bar{n})$. The short proof of $\Gamma \rightarrow B(\bar{n})$ corresponds to the 'purely number-theoretic' proof in Theorem and the proof in $L K$ of $A_{0} \rightarrow \forall x(B(x) \equiv A(x))$ corresponds to the proof in $L K$ in Theorem.

The reason why we cannot make the above relation rigorous is the fact that $\boldsymbol{S}_{\boldsymbol{o}}$ contains equations of the forms (11) and (12) in the definition of $\boldsymbol{S}_{\boldsymbol{o}}$.

REMark 2. Lemma 1 shows a proof-theoretic meaning of the notion 'diophantine'. Lemma 2 is a careful formalization of the proof given in [4] of MRDP theorem. Theorem shows a proof-theoretic meaning of MRDP theorem.

Remark 3. We can conclude that the affirmative answer to Problem in the introduction implies the negative answer to the k-provability problem of $\boldsymbol{P A}$, because the following proposition holds.

PROPOSITION. For each recursive enumerable subset $X$ of $\omega$, there exist a natural number $m$ and a formula $A(x)$ such that for each natural number $n$ the following propositions (1), (2) and (3) are equivalent.

(1) $n$ belongs to $X$.

(2) $\boldsymbol{L} \boldsymbol{K} \stackrel{m}{-} \boldsymbol{S}_{\mathbf{0}} \rightarrow A(\bar{n})$.

(3) $A(\bar{n})$ is provable in $P A^{*}$.

Proof. Take any recursive enumerable subset $X$ of $\omega$. Then, by MRDP theorem, there are polynomials $g\left(n, n_{1}, \cdots, n_{\nu}\right)$ and $h\left(n, n_{1}, \cdots, n_{\nu}\right)$ with natural 
number coefficients for which the following two propositions are equivallent.

(a) $n$ belongs to $X$.

(2) $g\left(n, n_{1}, \cdots, n_{\nu}\right)$ is equal to $h\left(n, n_{1}, \cdots, n_{\nu}\right)$ for some natural numbers $n_{1}, \cdots$, $n_{\nu}$

We can construct an E-formula $A(x)$ which expresses naturally the proposition (2) above. Now Proposition is immediate from (3) in Lemma 1 and the fact that each provable sentence in $\boldsymbol{P} \boldsymbol{A}^{*}$ is true.

\section{References}

[1] M. Baaz, Note on the existence of most general semi-unifiers, Arithmetic, proof theory and computational complexity (P. Clote and J. Krajičk, editors), Oxford Univ. Press, New York, 1993, pp. 20-29.

[2] M. Baaz and P. Pudlák, Kreisel's conjecture for $\mathrm{L}_{\exists}$, Arithmetic, proof theory and computational complexity (P. Clote and J. Krajicek, editors), Oxford Univ. Press, New York, 1993, pp. 30-60.

[ 3 ] S.R. Buss, The undecidability of k-provability problem, Annals Pure Appl. Logic, 53 (1991), 75-102.

[4] M. Davis, Hilbert's tenth problem is unsolvable, Amer. Math. Mon., 80 (1973), 233269.

[5] W.M. Farmer, A unification-theoretic method for investigating the k-provability problem, Annals Pure Appl. Logic, 51 (1991), 173-214.

[6] H. Gaifman and C. Dimitracopoulos, Fragments of Peano s arithmetic and the MRDP theorem, Logic and Algorithmic, Monograph. Enseign. Math. 30 (Enseignement Math.), Geneva, 1982, pp. 187-206.

[ 7] S.C. Kleene, Introduction to metamathematics, North-Holland, Amsterdam, 1952.

[ 8 ] J. Krajicek and P. Pudlák. The number of proof lines and the size of proofs in first order logic, Arch. Math. Logic, 27 (1988), 69-84.

[ 9 ] J. Krajicek, Generalizations of proofs, Proceedings of 5th Easter Conference on Model Theory, Humboldt Univ., Easter Berlin, 1987, pp. 82-99.

[10] G. Kreisel, Logical aspects of computation: contributions and distractions, Logic and computrr science (P. Odifreddi, Editor), Academic Press, New York, 1990, pp. 205-278.

[11] V.P. Orevkov, Reconstruction of a proof from its scheme, Soviet Math. Dokl., 35 (1987), 326-329.

[12] V.P. Orevkov, Lower bounds on the lengths of derivations in arithmetic in terms of the complexity of terms involved in the derivations, Soviet Math. Dokl., 35 (1987), 579582.

[13] V.P. Orevkov, Complexity of proofs and their transformations in axiomatic theories, Amer. Math. Soc., 1993.

[14] J. Paris and L. Harrington, A mathematical incompleteness in Peano arithmetic, Handbook of mathematical logic (J. Barwise, Editor), North-Holland, Amsterdam, 1977, pp. 1133-1142.

[15] D. Richardson, Sets of theorems with short proofs, J. Symbolic Logic, 39 (1974), 235242.

[16] G. Takeuti, Procf theory (second edition), North-Holland, Amsterdam, 1987.

[17] T. Yukami, Some results on Speed-up, Ann. Japan Assoc. Philos. Sci., 6 (1984), 195205 . 
[18] T. Yukami, Taking out LK-parts from a proof in Peano arithmetic, J. Symbolic Logic, 51 (1986), 682-700.

[19] T. Yukami, A theorem on generalization of proofs, Arch. Math. Logic, 30 (1990), 139153. 